\title{
Treatment of Pediatric Life Threatening Amitriptilin Intoxication with Plasma Exchange
}

\author{
Deniz Karakaya ${ }^{1 *}$, Oktay Perk $^{2}$, Serhan Özhan², Deniz Tekin ${ }^{1}$, Tanil Kendirli² \\ ${ }^{1}$ Department of Pediatric Emergency Department, Ankara University Medical School, Ankara, Turkey. \\ ${ }^{2}$ Department of Pediatric İntensive Care Department, Ankara University Medical School, Ankara, Turkey.
}

*Corresponding Author: Deniz Karakaya, Department of Pediatric Emergency Department, Ankara University Medical School, Ankara, Turkey.

Received date: June 07, 2021; Accepted date: August 02, 2021; Published date: Augusł 11, 2021

Citation: D Karakaya, O Perk, S Özhan, D Tekin, T Kendirli. (2021) Treatment of Pediatric Life Threatening Amitriptilin Intoxication with Plasma Exchange. International Journal of Clinical Case Reports and Reviews. 8(1); DOI:10.31579/2690-4861/143

Copyright: () 2021 Deniz Karakaya, This is an open-access article distributed under the terms of the Creative Commons Attribution License, which permits unrestricted use, distribution, and reproduction in any medium, provided the original author and source are credited.

\begin{abstract}
Tricyclic antidepressant (TCA) overdose is one of the most common causes of serious drug poisoning in children. Amitriptyline is a major TCA drug that is used widely. Tricyclic antidepressant intoxications are very important because of their severe adverse effects and probable fatal outcomes. It may cause cardiovascular, respiratory and neurological side effects. Poisoning results in hypotension, cardiac dysrhythmia, depression of the central nervous system (CNS) and seizures. The most common effects on the central nervous system are agitation, lethargy, seizures, and coma. Cardiovascular toxicities manifest itself especially with electrocardiographic (ECG) abnormalities, arrhythmias, and refractory hypotension and they are the leading cause of fatal outcome.

Treatments in TCA overdose are mainly conservative including gastric lavage, activated charcoal and vasopressors for hypotension, sodium bicarbonate for dysrhythmias, and benzodiazepines for seizures. Magnesium sulfate (MgSO4) also has an effective role in the treatment of fatal cardiac arrhythmias occurring in high-dose amitriptyline intoxication. Intravenous lipid emulsions have been increasingly studied as antidotes to reverse acute, life-threatening drug toxicity. Unpredictable and poor results with hemoperfusion (HP) and hemodialysis (HD) should be expected, as the drug binds rapidly to tissues and has a large volume of distribution. But beside it, in the last few years, HP has been successfully used in severe TCA overdose, especially in patients with persistent respiratory, cardiac, and neurologic symptoms. Besides all these, recently, plasma exchange, have been increasingly used. A reduction of plasma levels by $63 \%$ after plasmapheresis in TCA poisoning has been reported. Here, we report a successful treatment of plasma exchange 3 year patient with amitriptyline overdose who had arrhythmias and seizures that wasn't respond all to conservative therapies.
\end{abstract}

Keywords: amitriptyline; plasma exchange; intoxication; pediatric

\section{Introduction}

Tricyclic antidepressant (TCA) overdose is one of the most common causes of serious drug poisoning in children. Amitriptyline is a major TCA drug that is used widely. Tricyclic antidepressant intoxications are very important because of their severe adverse effects and probable fatal outcomes. It may cause cardiovascular, respiratory and neurological side effects. Poisoning results in hypotension, cardiac dysrhythmia, depression of the central nervous system (CNS) and seizures [1]. The most common effects on the central nervous system are agitation, lethargy, seizures, and coma. Cardiovascular toxicities manifest itself especially with electrocardiographic (ECG) abnormalities, arrhythmias, and refractory hypotension and they are the leading cause of fatal outcome [2].

Treatments in TCA overdose are mainly conservative including gastric lavage, activated charcoal and vasopressors for hypotension, sodium bicarbonate for dysrhythmias, and benzodiazepines for seizures [1]. Magnesium sulfate (MgSO4) also has an effective role in the treatment of fatal cardiac arrhythmias occurring in high-dose amitriptyline intoxication $[3,4]$. Intravenous lipid emulsions have been increasingly studied as antidotes to reverse acute, life-threatening drug toxicity $[5,6]$. Unpredictable and poor results with hemoperfusion (HP) and HD should be expected, as the drug binds rapidly to tissues and has a large volume of distribution $[7,8,9]$. But beside it, in the last few years, HP has been successfully used in severe TCA overdose, especially in patients with persistent respiratory, cardiac, and neurologic symptoms [10, 11]. Besides all these, recently, plasma exchange $[12,13]$, have been increasingly used. Plasma exchange is the removal of the patient's plasma and replacement with another fluid. A reduction of plasma levels by $63 \%$ after 
plasmapheresis in TCA poisoning has been reported [14]. Here, we report a successful treatment of plasma exchange 3 year patient with amitriptyline overdose who had arrhythmias and seizures that wasn't respond all to conservative therapies.

\section{Case report}

A 3, 5-year-old girl was brought with 112 ambulance complaints of wheezing, bruising, blurring of consciousness and seizures. We learned that the patient slept at 22:00 in the evening and she woke up at 3:00 in the morning when the family noticed the wheezing breath of the patient. When the parents notice her, she was cyanotic, the respiration was irregular and the consciousness was closed. She delivered to our unit by 112 emergency servise. A brief (5-10 sc) generalized tonic clonic seizure was observed 3 times during the transfer of the patient. The patient's resume and family trait did not have a feature.

On physical examination, heart rate was $110 / \mathrm{min}$, respiration rate $32 / \mathrm{min}$ ,blood pressure 110/60 mm Hg SPO2: 99\% temperature: $36.1 \mathrm{C}$ and blood sugar: $118 \mathrm{mg} / \mathrm{dL}$. Overall status: Poor Neurological: Consciousness and her response to painful stimulus was minimal DTR: normoactive IR: Bilateral weak and MI: assessed as absent. Respiratory irregularities and coarse secretary breath sounds were present. Other system examinations were natural. Total blood count: normal, the electrolyte levels, liver and kidney function tests were normal. Acute phase reactants negative, blood gas was $\mathrm{pH}: 7.27$ pCO2: 32 lac: 1.7 hCO3: 15.5 coHB: 0.8 .

The patient was monitorized. Intravenous route opened. She was initially given $20 \mathrm{ml} / \mathrm{kg}$ isotonic solution for circulatory support. Her respiration was stable and effective so she was not intubated at that time. Short seizures and consciousness blurring were assessed as continuing patient status. The patient was given IV diazepam two times. Intravenosus eptantoin and iv levatiracetam were loaded. The patient's convulsions stopped but the consciousness was closed. The story of the patient, who was completely healthy before, was repeated. Her relatives didn't estimated that she had taken medicine or travma. Computed tomography was normal. There was no height of fever. There was no epilepsy story in the family. The patient was interrogated for drug intake again. The family said they saw a medication box in the hands of the patient at noon the day before, but did not know what the medicine was. We wanted to bring the medicine box from the family. At that time, extrasystoles were identified on the monitor. Learned that the drug is laroxyl (amitriptyline) and the treatment and follow-up of the patient was approached as TCA intoxication. The patient weight was $15 \mathrm{~kg}$. It was estimated that she took approximately $35 \mathrm{mg} / \mathrm{kg}$ of amitriptyline about 18 hours before admission. A sodium bicarbonate IV bolus was administered for the patient's extrasystoles. The patient was observed to return to sinus rhythm for a short time. For this reason, $\mathrm{NaHCO} 3$ was repeated 5 times and followed by continuous IV infusion for treatment of cardiac arrhythmias and for alkalinization. Patient received intensive care. Lidocaine therapy for arrhythmia was started. Because the patient's clinical condition did not improve, hemoperfusion was considered. Plasma Exchange was done. On the follow-up, the patient's consciousness was opened, the arrhythmia was strained, the seizure did not recur. Support treatments were cut off. On the second day of admission, the patient did not need intensive care and was discharged after 24 hours of more observation.

\section{Discussion}

Amitriptyline is registered as a tricyclic antidepressant (TCA). It is also being used off label for additional purposes, such as neuropathic pain, tension headache, and posttraumatic stress. Tricyclic antidepressant overdosage has toxic effects over cardiovascular, autonomous nervous, and central nervous systems, and may result in cardiac conduction delays, dysrhythmia, hypotension, altered mental status and seizures [14]. Serious and lethal toxic effects have been reported with TCA intoxications of 10 to $30 \mathrm{mg} / \mathrm{kg}$ and $50 \mathrm{mg} / \mathrm{kg}$, respectively $[1,14]$. Exact fatal serum levels of amitriptyline in children are not known. Ash et al. reported that the lethal dose can be variable and ingestion of more than $1 \mathrm{~g}$ TCA or a blood level of $1,000 \mathrm{mg} / \mathrm{l}$ predicts a high risk of mortality [10]. Children are more susceptible to TCA overdose and fatal cases with ingestion of only one capsule or table have been reported [14]. For this reason, estimated amount of drug taken based on anamnesis alone may be misleading.

Conduction delays may become apparent on electrocardiogram as PR and QT prolongation, QRS widening that may resemble right bundle block or Brugada electrocardiographic pattern $[15,16]$. Widening of the QRS complex> 100 milliseconds is associated with the development of seizures and arrhythmias. Sinus and supraventricular tachycardia, premature ventricular complexes, idioventricular tachycardia, VT or fibrillation may be seen $[1,14]$. The most serious causes of death in TCA intoxication are persistent hypotension. In addition that, possible mechanisms for TCA-induced neurotoxicity include g-aminobutyric acid receptor antagonism, neuronal $\mathrm{Na}$ channel blockage and central anticholinergic activity [5].

Recommended treatments in TCA overdose are mainly conservative, including gastric lavage and activated charcoal and bicarbonate infusion to prevent cardiac side effects $[17,18]$. Inhibition of absorption by gastric lavage should be considered if ingestion was recent (1-2 hours) and activated charcoal can be administered because amitriptyline delays gastric emptying due to its anticholinergic effects. Repeated doses of charcoal may be necessary to interrupt the enterohepatic cycle. In Tricyclic antidepressant intoxication, sodium bicarbonate treatment is suggested as the first agent. Sodium bicarbonate is known to decrease ventricular arrhythmia frequency, prevent QRS prolongation, and correct hypotension. This effect can be accentuated by increasing plasma sodium concentration and alkalinization [14]. A decrease in Na entry into cells decreases the myocardial contractility and leads to hypotension [5]. Increasing plasma sodium concentration may prevent cardiac arrhythmias by decreasing sodium channel inhibition caused by TCA intoxication [1, 6]. Magnesium sulfate is also efficient in treating sustained ventricular arrhythmias, especially ventricular and torsade de pointes tachycardia and is known to prevent sudden deaths. Magnesium infusion is reported to increase intracellular potassium levels more significantly than potassium infusion. Antiarrhythmic and anticonvulsive agents are also used in symptomatic treatment. Benzodiazepine is the first choice for anticonvulsive therapy and phenytoin is contraindicated for TCA-like effects. In the case of antiarrhythmic treatment, lidocaine should be preferred instead of the commonly used amiadorone.

Following oral administration, TCA are absorbed rapidly and peak plasma levels occur within 2-4 h [19]. Tricyclic antidepressant plasma elimination half-life is about $16 \mathrm{~h}$ in healthy volunteers ingesting a single therapeutic dose. Ash et al. Tricyclic antidepressants bound to plasma proteins with high affinity [10]. Moreover, these drugs are not dialysable because of their high protein affinity. Frank and Klerdorf reported that HD would only compensate the acid-base and electrolyte status however, there are conflicting reports of the efficacy of HP in TCA poisoning [20]. Similarly, Bayrakc, i et al. reported successful treatment of severe amitriptyline poisoning in three cases by plasma exchange [21]. After ingestion, TCA are absorbed rapidly and quickly taken up by the tissues, importantly by the lung and the heart [10]. It seems reasonable that HP performed in an early stage of ingestion would be more efficient [8]. In addition to removing free drug in blood, removal of unmeasured metabolite from the blood could also help in clinical improvement [10]. Heath et al. reported that respiratory depression, seizure severe arrhythmias such as ventricular tachycardia or fibrillation, or an increased QRS interval are the criteria for HP use in TCA overdose [7]. There is also an increasing interest in the use of intravenous lipid emulsion therapy to treat life threatening toxicity associated with lipophilic drugs in adults 
and children. In the 14 pediatric cases we reviewed, 13 patients appeared to have a beneficial response following intravenous lipid emulsion therapy, despite being unresponsive to standard resuscitation methods [22]. Further reporting of pediatric cases will guide the optimal use and safety profile of intravenous lipid emulsion for the emergent treatment of acute drug toxicity [22].

In our case we experienced that, the detailed questioning of the history is very important. Our patient was initially assessed as status. The treatment approach then changed completely. Since our patient was admitted approximately 18 hours after drug ingestion, gastric lavage was not performed. The patient who was conscious off was not given active charcoal. The first drug that we administered to our patient was sodium bicarbonate infusion, but it did not make totally change in the cardiac rhythm. İntravenous lidocain was initiated for arrytmies. We did not record a marked decrease in our patient's blood pressure. It was either because she did not have hypotension or having been given intravenous isotonic crystalloid solution from the time of admittance. Phenytoin loading was performed for the patient's seizure. Phenytoin was contraindicated in these patients as an antiepileptic agent. This might have triggered arrhythmia. The anticonvulsant treatment plan could change if the etiologic agent was learned earlier. Despite the conservative and symptomatic treatment, our patient continued to have persistent symptoms. Then HP was considered to control the resistant arrytmies and unconsciousness. The arrytmies returned to normal sinus rhythm at the end of HP and his seizures did not recurr. We observed a very good clinical response in our patient and found HP a useful treatment modality in a child with severe amitriptyline intoxication.

\section{References}

1. Citak A, Soysal DD, Ucsel R, et al. (2002) Efficacy of long duration resuscitation and magnesium sulphate treatment in amitriptyline poisoning. Eur J Emerg Med. 9:63-66.

2. Bailey DN, Van Dyke C, Langou RA, et.al. (1978) Tricyclic antidepressants: plasma levels and clinical findings in overdose. Am J Psychiatry. 135:1325-1328.

3. Heard K, O'Malley GF, Dart RC. (1999) Treatment of amitriptyline poisoning with ovine antibody to tricyclic antidepressants. Lancet. 354:1614-1615.

4. Thanacoody HK and Thomas SH. (2005) Tricyclic antidepressant poisoning: cardiovascular toxicity. Toxicol Rev. 24: 205-214.

5. Mills K., Brent J, Wallac KL, et al. (2005) Critical care toxicology: diagnosis and management of the critically poisoned patient. Philadelphia. 475-484.

6. Knudsen K, Abrahamsson J. (1994) Effects of epinephrine, norepinephrine, magnesium sulfate, and milrinone on survival and the occurrence of arrhythmias in amitriptyline poisoning in the rat. Crit Care Med. 22:1851-1855.
7. Heath A, Wicksstr_m I, Martensson E, et al. (1982) Treatment of antidepressant poisoning with resin hemoperfusion. Human Toxicol. 1:361-371.

8. Diaz-Buxo JA, Farmer CD, Chandler JT. (1978) Hemoperfusion in the treatment of amitriptyline intoxication. Trans Am Soc Artif Intern Organs. 24:699-703.

9. Ryan R, Wians FH, Stigelman WH, et al. (1985) Imipramine poisoning in a child: lack of efficacy of resin hemoperfusion. Pediatr Emerg Care. 1:201-204.

10. Ash SR, Levy H, Akmal M, et al. (2002) Treatment of severe tricyclic anti depressant overdose with extracorporeal sorbent. Adv Ren Replace Ther. 9:31-41.

11. Bek K, Ozkaya O, Mutlu B, et al. (2008) Charcoal haemoperfusion in amitriptyline poisoning: experience in 20 children. Nephrology. 13: 193-197.

12. Jamaty C, Bailey B, Larocque A, et al. (2010) Lipid emulsions in the treatment of acute poisoning: a systematic review of human and animal studies. Clin Toxicol. 48:1-27.

13. Cave G, Harvey M, Graudins A. (2011) Review article: intravenous lipid emulsion as antidote: a summary of published human experience. Emerg Med Australas. 23:123-141.

14. Geis GL, Bond GR. (2005) Antidepressant overdose: tricyclics, selective serotonin reuptake inhibitors, and atypical antidepressants. Pediatric Toxicology: Diagnosis and Management of the Poisoned Child. 1st ed. New York, NY: The McGraw-Hill Companies. 296-302.

15. Harrigan RA, Brady WJ. (1999) ECG abnormalities in tricyclic antidepressant ingestion. Am J Emerg Med. 17:387-393.

16. Bebarta VS, Phillips S, Eberhardt A, et al. (2007) Incidence of Brugada electrocardiographic pattern and outcomes of these patients after intentional tricyclic antidepressant ingestion. Am J Cardiol. 100: 656-660.

17. Liebelt EL. (1998) Targeted management strategies for cardiovascular toxicity from tricyclic antidepressant overdose: the pivotal role for alkalinization and sodium loading. Pediatr Emerg Care. 14: 293-298.

18. Haddad LM. (1992) Managing tricyclic antidepressant overdose. Am Fam Physician. 46: 153-159.

19. Amsterdam J, Brunswick D, Mendels J. (1980) The clinical application of tricyclic antidepressant pharmacokinetics and plasma levels. Am J Psychiatry. 137:653-662.

20. Frank RD, Kierdorf. (2000) HP Is there a role for hemoperfusion/hemodialysis as a treatment option in severe tricyclic antidepressant intoxication. Int $\mathrm{J}$ Artif Organs. 23:618-623.

21. Bayrakc,i B, Unal S, Erkoc,og ơlu M, et al. (2007) Case reports of successful therapeutic plasma: exchange in severe amitriptyline poisoning. Ther Apher Dial. 11:452-454.

22. Joseph D Presley, Peter A Chyka. (2013) Intravenous Lipid Emulsion to Reverse Acute Drug Toxicity in Pediatric Patients. Ann Pharmacother. 47(5):735-743. 